\title{
基于可穿戴传感器数据的人体行为识别数据特征提取方法
}

\author{
郭毅博, 孟文化 ${ }^{*}$, 范一鸣, 侯立硕, 袁玥, 薛均晓, 徐明亮 \\ (郑州大学信息工程学院 郑州 450001) \\ (mengwenhuazzu@gmail.com)
}

\begin{abstract}
摘 要: 随着可穿戴设备的发展与普及, 基于可穿戴传感数据进行人体行为检测展现了巨大的研究价值. 目前大多 人类行为识别工作都是基于视频图像展开的, 然而, 使用计算机视觉技术进行人类行为识别存在 2 个挑战: 一是很难 使参与数据采集的人员在自然状态下采集真实状态下的运动数据, 在开展数据采集之前往往需要对参与数据采集的 人员进行培训并严格规范其采集动作, 最终得到的数据将是背离真实生活的数据, 其研究价值将大打折扣; 二是数 据采集过程中还涉及采集人员的隐私保护问题. 为此, 提出一种基于深度学习的数据特征提取方法. 首先在灵活设 置卷积核的基础上引人神经网络的分支结构多尺度提取原始数据的深度特征; 然后将各分支得到的数据特征进行融 合并作为下一个卷积层的输人. 实验结果表明, 与目前主流方法相比, 该方法在 MHEALTH, WHARF 和 USCHAD 这 3 个标准数据集上的准确率和召回率都取得了更好的效果. 此外, 该方法还在 2 个较新数据集 Stanford-ECM Dataset 和 DATAEGO 上做了验证, 结果表明该方法具有较好的泛化能力.
\end{abstract}

关键词：卷积神经网络; 可穿戴设备; 卷积核; 分支网络

中图法分类号: TP391.41 DOI: 10.3724/SP.J.1089.2021.18690

\section{Wearable Sensor Data Based Human Behavior Recognition: a Method of Data Feature Extraction}

\author{
Guo Yibo, Meng Wenhua ${ }^{*}$, Fan Yiming, Hou Lishuo, Yuan Yue, Xue Junxiao, and Xu Mingliang \\ (Information Engineering School, Zhengzhou University, Zhengzhou 450001)
}

\begin{abstract}
With the development of wearable devices, it is great research value to conduct human behavior detection based on wearable sensor data. At present, most human behavior recognition work is based on images. However, there are two challenges in using computer vision technology for human behavior recognition. Firstly, it is difficult to make users involved in the data collection in the nature under the true state of motion data, before starting data collection, it is often necessary to train the personnel involved in data collection and strictly regulate their collection actions. Data that collected in that way will be a departure from the real life. Its research value will be discounted. Secondly, the privacy protection of data collectors is involved in the process of data collection. To this end, a data feature extraction algorithm is proposed based on deep learning. Firstly, introduces the branch structure of neural network on the basis of flexible convolution kernel setting to extract the depth characteristics of the original data at multiple scales. After that, the data features obtained by each branch are fused and used as the input experiment of the next convolution layer.

收稿日期：2020-09-30; 修回日期：2020-11-17. 基金项目：国家自然科学基金(61602421); 中国博士后基金(2016M600584). 郭毅博(1986-), 男, 博士, 讲师, 硕士生导师, CCF 会员, 主要研究方向为人工智能; 孟文化(1993一), 男, 硕士研究生, 论文通讯作 者, 主要研究方向为计算机视觉、深度学习; 范一鸣(1996一), 男, 硕士研究生, 主要研究方向为计算机视觉; 侯立硕(1996一), 女, 硕 士研究生, 主要研究方向为深度学习; 袁玥(1996一), 女, 硕士研究生, 主要研究方向为数据可视化; 薛均晓(1982一), 男, 博士, 硕 土生导师, CCF 会员, 主要研究方向为人工智能、虚拟现实; 徐明亮(1981一), 男, 博士, 教授, 博士生导师, CCF 会员, 主要研究方向 为计算机图形学、人工智能.
\end{abstract}


The experimental results show that compared with the current mainstream algorithm, proposed algorithm has achieved better results in accuracy and recall rates on the three standard data sets of MHEALTH, WHARF and USCHAD. In addition, the method has been verified on two newer data sets, Stanford-ECM Dataset and DATAEGO Dataset, and the results show that the method has good generalization ability.

Key words: convolutional neural networks; wearable devices; convolution kernel; branch

随着社会的快速发展、人类寿命的增长以及生 育观念的转变, 我国人口老龄化问题越发突出, 由 此引发的空巢老人的健康安全问题已受到社会各 界的广泛关注. 通过加速度计、心率计等传感器收 集的传感数据开展人体行为检测对保障老人，尤 其空巢老人的日常安全具有重要的应用价值. 基 于可穿戴设备的人体行为检测 ${ }^{[1]}$ 已成为当前热门 的研究课题, 其中一个主要问题是如何有效地将 可穿戴传感器收集的传感数据映射到人类的行为 活动. 在长期收集人体行为数据后, 可对人体健康 进行有效监控. 具体地, 该行为记录可在患病状况 下, 辅助医生制定更加科学的治疗方案并提出稳 妥的康复锻炼建议; 另外, 也可针对个体定制更加 合理的锻炼计划.

目前，大多人体行为识别都是基于视频图像 展开的. 为保证充足、高质量的视频图像数据，传 统的基于视频图像的人体行为识别方法 ${ }^{[2-4]}$ 往往需 要在一个固定区域内安装大量摄像头, 即便如此, 其采集图像时也无法实现对用户全时段无间断跟 拍; 同时, 数据采集前需要脚本化培训数据采集人 员，这导致与真实生活状态相差甚远，无法记录个 体真实的行为数据. 此外，在实际采集图像信息过 程中，隐私保护问题也是一大挑战. 随着可穿戴设 备的智能化发展, 加速度计、磁力计、心率计等传 感器被内置在智能手机和智能手环中, 可长时间 跟踪采集人体行为数据，并充分保护个人隐私. 基 于可穿戴设备的人体行为检测方法, 用户只需佩 戴自己的手机以及智能手环就可以完成数据的采 集. 这种数据采集方法的优势如下：(1) 数据采集 场景多样化. 不受环境、天气等外部条件的限制. (2) 数据真实. 可以在自然状态下记录一个人全时 段的完整的运动数据. (3) 数据集完整. 与视频采 集相比, 采用可穿戴智能设备(智能手机、智能手 环)采集数据不受时间、地点以及人为约束.

可穿戴设备传感数据的人体行为方法已取得 丰富的研究成果. 为了进一步提升基于可穿戴设 备传感数据的行为识别有效性，本文在 Chen 等 ${ }^{[5]}$
和 $\mathrm{Ha}$ 等 ${ }^{[6]}$ 工作的基础上提出一种基于深度学习的 数据特征提取方法，在灵活设置卷积核的基础上， 引人神经网络的分支结构多尺度提取原始数据的深 度特征, 将各分支得到的数据特征进行融合并作为 下一个卷积层的输人. 实验结果表明, 与目前主流 方法相比, 本文方法在 MHEALTH ${ }^{[7]}, \mathrm{WHARF}^{[8]}$, $\mathrm{USCHAD}^{[9]}$ 这 3 个标准数据集上, 无论是准确率还 是召回率都取得了更好的效果. 同时在 2 个较新的 数据集 Stanford-ECM Dataset ${ }^{[10]}$ 和 DATAEGO ${ }^{[11]}$ 上进行验证, 实验表明本文方法具有较好的泛化 能力. 此外, 将本文方法引人零样本识别任务中, 也取得了较好的效果。

\section{1 相关工作}

基于可穿戴设备传感数据进行人体行为识别 的过程如图 1 所示. 首先通过可穿戴设备(智能手 表、智能手机)采集人体的加速度、心率等数据, 并 以固定的时间窗口扫描原始数据获得样本作为识 别模型的输人; 然后采用人体行为识别模型识别 其具体行为类别. 获得各个行为类别后, 生成个体 行为数据集.

\section{1 可穿戴设备与人体行为识别}

可穿戴设备最早出现在 20 世纪中期, 它不仅 是一种硬件设备, 还能够进行数据交互, 使用者通 过佩戴具有特定功能的电子设备记录自己的日常 行为或身体健康状况. 传统的方法是把传感器直 接佩戴在用户身上, 收集用户的动作数据. 随着智 能设备的发展, 研究人员普遍选择把传感器嵌人 移动设备. 例如, 路知远 ${ }^{[12]}$ 将传感器嵌人随身医 疗设备中, 设计了一款新的脉搏血氧仪, 实时监测 老人的身体状况. 对于可穿戴传感器人体行为检 测的复杂行为识别, 一般生活中的复杂行为可以 通过各类基本动作组合来表现，如看书就包括翻 书、看等几个基本动作. 汪亮 ${ }^{[13]}$ 通过引人 Emerging Pattern 模型识别复杂行为. 茹晨光 ${ }^{[14]}$ 采用分层行 为识别的方法提升移动设备对设备位置变化的适 


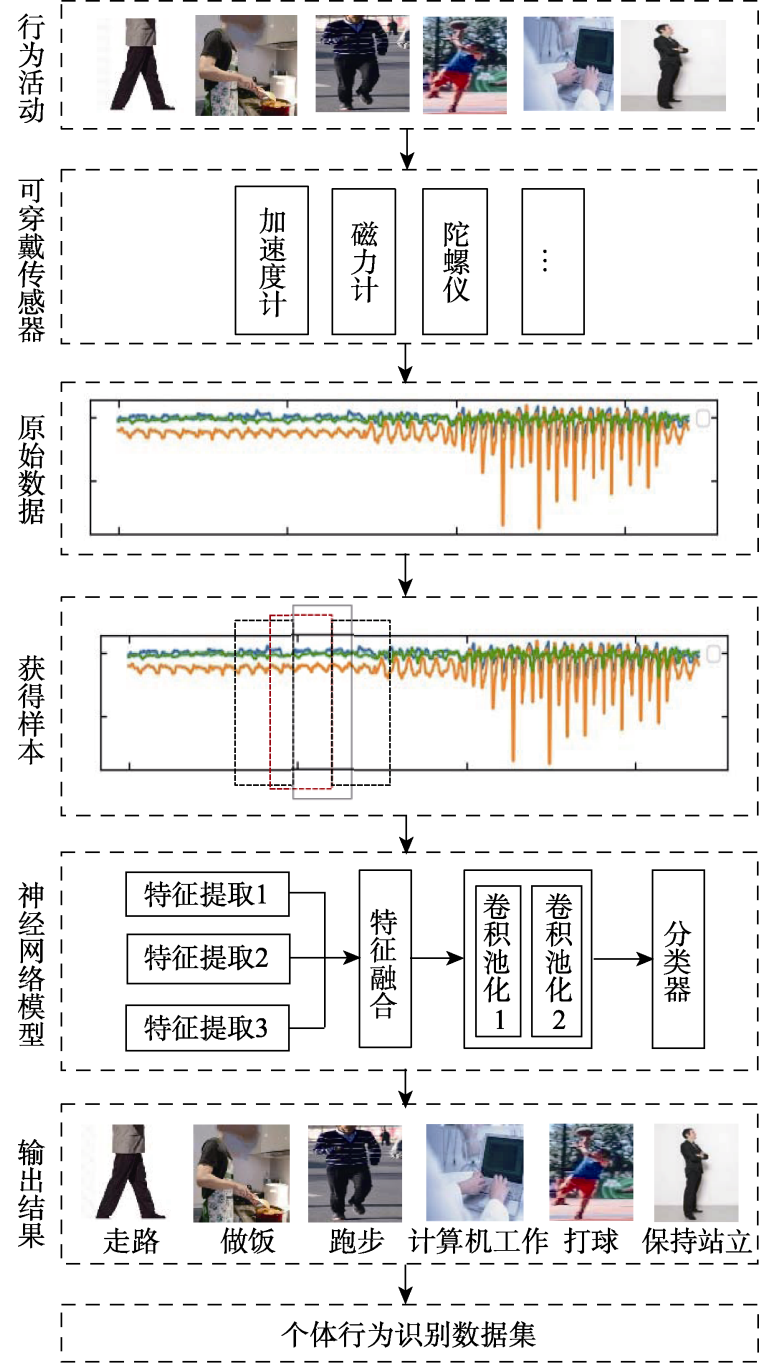

图 1 基于可穿戴传感设备的行为识别过程

应能力, 最终准确率提升到 $93.300 \%$. 在复杂行为 识别方面, Amato 等 ${ }^{[15]}$ 考虑了人与人、人与周围环 境之间的交互, 利用分布在人和物上的传感器收 集行为数据, 提高对复杂行为的识别.

\section{2 人体行为识别}

在人体行为识别中, 最具代表性的算法是 2016 年 Ohnishi 等 ${ }^{[16]}$ 提出的改进的稠密光流轨迹 (improved dense trajectories, iDT)算法, 它是一种 基于光流的稠密光流轨迹(dense trajectories, DT) ${ }^{[17]}$ 算法, 在卷积神经网络未应用于人体行为检测之前 是效果最好的行为检测算法. 随着卷积神经网络的 发展, Simonyan 等 ${ }^{[18]}$ 在 2014 年提出 Two-stream 方 法，在视频的空间上和时间上进行行为特征的提 取. 与 iDT 相比, Two-stream 方法的鲁棒性更高. 2015 年, Tran 等 ${ }^{[19]}$ 提出的三维卷积(convolutional 3D, C3D)方法可以直接处理视频，在时空特征提 取上非常有效, 该方法提升了检测速度，但准确率
较低. 2016 年, Wang 等 ${ }^{[20]}$ 提出时间感知网络(time sensitive networks, TSN), 通过对长视频进行分段 处理，解决了 Two-stream 方法中无法对长时间结 构建模的问题，但 TSN 方法的结构复杂，检测速 度慢. 2017 年, Lan 等 ${ }^{[4]}$ 在 TSN 方法的基础上提出 提取局部视频特征(deep local video feature, DLVF) 的方法, 针对 TSN 中不同片段分配不同的权重, 更 加合理地对不同片段的判定结果进行融合. 2020 年, 丁重阳等 ${ }^{[21}$ 提出一种基于时空权重姿态运动特征 的行为识别方式, 通过动态时间规整和傅里叶时 间金字塔算法建模，最终提高了人体行为识别的 精确度.

\section{2 数据分析}

本文中的传感数据包含加速度数据、心率数 据、陀螺仪数据等，它们在人类不同行为活动中的 数据特征有明显的区别. 在绝大多数情况下, 跑步 和走路的心率数据存在显著差异，原因是对于同 一个人, 相对于走路, 跑步过程中需要消耗大量的 氧气和能量, 所以肺部需要尽快吸进一些氧气, 心 脏活动会加快, 血液循环加速运输营养物质和氧 气; 但经常参加体育运动与不常运动的人相比, 其 心跳加快等特征不明显. 因此, 基于单模态心率数 据特征的识别模型在行为识别任务中对部分人群 效果不好. 此外, 由于人与人之间体质的差异, 每 个人在走路和跑步时的心率浮动区间存在客观差 异. 为补齐心率数据在行为检测任务中的不足, 适 应更多的人群, 研究人员引人更多模态的传感数 据, 如加速度数据(加速度数据并不是真正的加速 度, 而是传感器位置的一系列的三维坐标). 文献 [9]研究表明, 加速度数据在人类行为检测方面具 有独特优势, Bruno 等 $^{[8]}$ 发布了只包含加速度数据 的数据集, 并在其数据集上验证了这一观点. 本文 通过分析发现, 在不同活动方式之间加速度数据 存在显著差异. 如图 2 所示, 走路和跑步的加速度 数据在 $x, y, z$ 这 3 个轴上的区分度都非常大，人在 从走路到跑步的过程中数据在三维空间内幅度变 化显著, 这对于区分活动类别具有很大的正向促 进作用.

\section{3 网络设计}

在神经网络设计过程中，尤其是基于可穿戴 


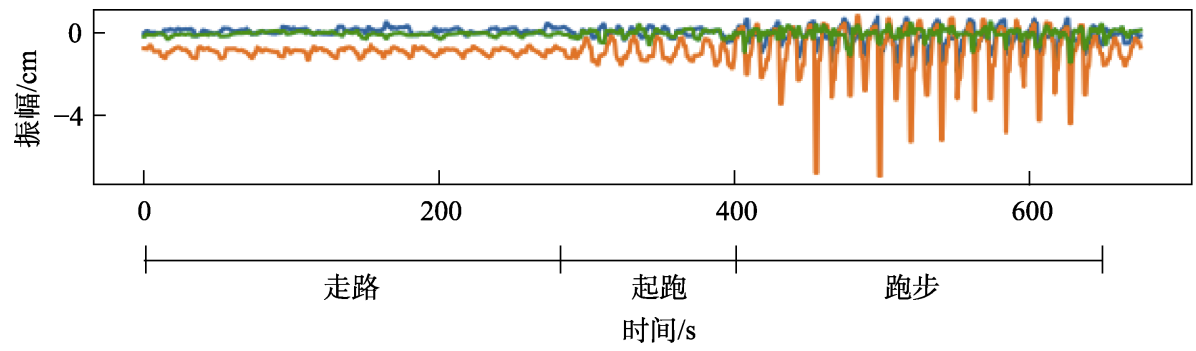

图 2 走路到跑步过程的加速度变化曲线

传感数据的人类行为检测任务中, 卷积核的设置 尤为重要. Chen 等 ${ }^{[5]}$ 对此做出了非常充分的研究与 论证, 证明了卷积核设置的重要性. Ha 等 ${ }^{[6]}$ 在设计 神经网络结构时引人分支结构, 即对不同传感器 来源数据分别使用一个分支网络单独提取特征, 然后将它们融合, 再经过一系列卷积池化操作之 后输人分类器.

文献[5-6]设计的网络都有其显著优势，但也 有一定的不足之处. 其中, Chen 等 ${ }^{[5]}$ 对卷积核的设 计比较合理，但是忽略了不同数据间的关联特征; $\mathrm{Ha}$ 等 $^{[6]}$ 虽然引人了分支结构, 但忽略了不同传感 器来源数据之间的关联性. 综合 2 篇文献的优势并 改进其不足, 本文提出一个如图 3 所示的网络, 该 网络在每个分支上设置不同的卷积核以提取不同 维度的数据特征.

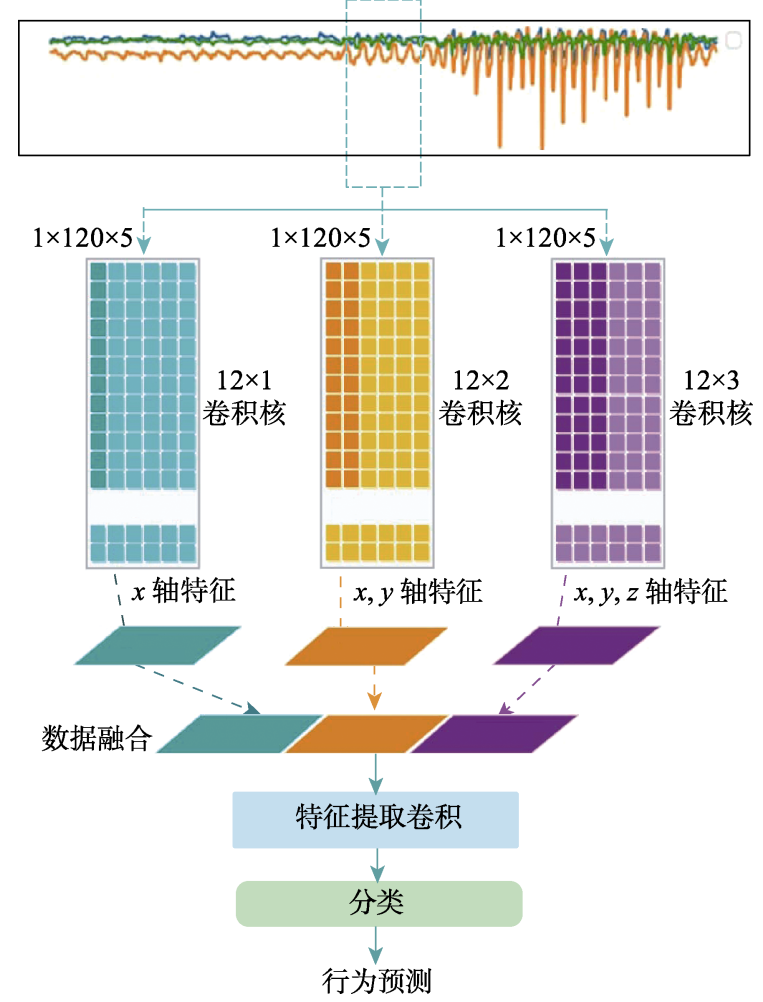

图 3 网络结构图

\section{1 卷积核的设置}

数据来源为加速度传感器、心率传感器、陀螺 仪等非图像类传感器时, 数据结构通常比较简单. 以加速度传感器和心率传感器为例, 加速度传感 器获的数据为三维坐标, 数据格式为 $(x, y, z)$; 心 率传感器数据更加简单, 为一维数据. 因此, 卷积 核的设置需考虑到不同传感数据之间的差异性以 及不同传感数据之间的关联. Chen 等 ${ }^{[5]}$ 的网络设计 过程中, 第 1 个卷积层使用的是大小为 $(12,1)$ 的卷 积核，其余各层的卷积核大小均为 $(12,3)$. 这种卷 积核的设置虽然在一定程度上可以保留数据特征 的独立性, 但是不能够更好地挖掘数据间的深层 次关联特征.

基于以上分析，本文设计的识别网络在卷积 核设计上延续了 Chen ${ }^{\text {等 }}{ }^{[5]}$ 的思想, 在其基础上多 尺度设计卷积核并添加分支结构, 目的是既能够 保持数据特征的独立性, 又能够挖掘数据间的关 联性. 如图 3 所示, 自左至右的 3 个分支结构分别 为分支 1 、分支 2 、分支 3 ，在分支 1 中，卷积核设 置为 $(12,1)$, 该分支设计的目的是挖掘单轴数据的 深层次特征; 分支 2 的设计原则是挖掘邻轴之间的 关联信息, 如需要挖掘 $x$ 轴和 $y$ 轴以及 $y$ 轴和 $z$ 轴 数据间的关联信息; 设计分支 3 的目的是在充分挖 掘某单一传感器数据整体特征的同时, 也能考虑 到与其他传感器数据的关联特征, 即该分支不仅 可以挖掘不同传感数据间的关联特征，而且能够 挖掘同一传感器数据(如加速度数据一三维坐 标)内所包含的 3 个轴之间的关联信息.

\section{2 分支结构}

分支结构具有显著优势，Ha 等 ${ }^{[6]}$ 在网络设计 中引人分支结构, 与其他方法相比取得了极佳的 效果. 该方法虽然引人了分支结构, 但是其卷积核 设置与其分支结构不能完全匹配; 且引人分支结 构的目的是对来自不同传感器的传感数据分别提 取数据特征, 忽略了数据之间的关联性. 此外, 其 分支结构设置比较复杂, 且未考虑数据的独立性. 
本文对 $\mathrm{Ha}$ 等 ${ }^{[6]}$ 方法扬长避短, 将分支结构引人本 文的网络设计; 在网络结构设计中引人 3 个分支, 每个分支结合其卷积核的设置具有不同作用, 如 图 3 所示, 分支 1 的作用是保持所有传感器数据的 单轴数据的独立性并挖掘其深度数据特征; 分支 2 的作用是挖掘不同轴 $(x, y, z)$ 数据的关联特征; 分支 3 的作用是挖掘不同传感器数据之间的关联 信息以及单个传感器(如加速度计)数据的特征. 将 通过 3 个分支得到的数据特征进行拼接, 再次进行 卷积操作, 最后进行分类.

\section{3 特征融合}

数据经过分支 1 分支 3 得到特征 $f_{1}, f_{2}, f_{3}$. 由于维度上不能完全匹配, 而它们的通道数、长度 是一样的, 因此在进行融合过程中, 不能采取传统 的点乘、叉乘、求和等特征融合方式. 根据 3 个特 征 $\left(f_{1}, f_{2}, f_{3}\right)$ 的实际情况，本文进行特征融合时采 取拼接的方式, 即将 3 个特征进行并列式拼接. 但 是, 这种拼接的特征融合方式是比较粗粘的, 为弥 补这种融合方式存在的不足，本文在完成特征拼 接之后将拼接好的特征输人卷积神经网络进行二 次特征提取. 经过二次特征提取实现对组合特征 的再处理，使三者能够实现深层次的融合并进一 步提升特征的质量, 经过二次特征提取得到的特 征输人分类器进行分类.

\section{4 本文方法}

图 3 所示的网络比较依赖数据的质量, 即数据 分布必须在一个合理的区间. 如常见的 ImageNet, Places Database 等经典的分类数据集的类别标签 都大致为均匀分布, 但当样本分布出现特别不平 衡或样本量不足的情况时, 该网络可能会对部分 类别存在过拟合等问题, 为此, 本文通过扩充样本 的方式解决该问题.

在开始训练模型之前, 需用 $N P_{i}$ (其中, $N$ 为样 本总数, $P_{i}$ 为第 $i$ 类样本在所有样本总数中的比 例)计算每类样本分布, 进行统计分析.

本文方法步骤如下。

输人. 样本分布信息 $\left\{N P_{1}, N P_{2}, \cdots, N P_{N}\right\}$.

输出. 新样本.

Step1. 依次读人各类样本分布信息, 如果 $N P_{i}>0.4$, 直接进行识别; 否则, 执行下一步.

Step2. 扩充样本量不足的类别数据, 若样本数量 不足, 则分别使用文献[22-23]方法合成样本来进行数据
增广。

Step3. 用图 3 所示的分类网络进行分类.

该方法的目的是克服数据集分布不平衡的问 题. 首先计算训练集的每类样本分布，对于某一类 别稀缺的样本, 通过数据增广、添加噪声及数据合 成的方式扩充数据; 当数据类别大致均匀分布时, 再通过本文构造的网络进行分类.

\section{5 实 验}

基于可穿戴设备采集的数据集，在人类行为 识别领域具有代表性的 MHEALTH ${ }^{[7]}$, WHARF ${ }^{[8]}$ 和 $\mathrm{USCHAD}^{[9]}$ 数据集进行实验, 以体现本文方法的 整体性能. 为了更好地说明本文方法的优势, 在 2 个比较新的数据集上 Stanford-ECM Datase ${ }^{[10]}$ 和 DATAEGO $^{[11]}$ 上也进行实验，其中，Stanford-ECM Dataset 数据集是 2017 年公开的, DATAEGO 数据 集是 2018 公开的. 由于数据集可能存在样本分布 不平衡等问题, 而且这些问题可能会造成模型过 拟合, 因此有必要通过实验证明模型性能的提升 是否是由于过拟合造成的. 本文也在 StanfordECM Dataset 数据集的基础上进行实验, 并分析其 性能提升的原因.

为了验证本文方法的通用性，本文还将该网 络用于零样本学习任务的分类部分, 即使用 Wang 等 ${ }^{[24]}$ 方法将可见类与未知类之间的语义关系参数 和可见类的原型特征合成未知类的虚拟原型特征 作为本文网络模型的输人, 输出为每个类别的语 义属性，根据语义属性判定类别. 这部分实验是在 CUB 数据集 ${ }^{[25]}$ 上展开的, 并与 Wang 等 ${ }^{[24]}$ 方法进 行比较.

\section{1 实验评价指标}

本文实验中采用 3 个评价指标——精度 $A$ 、召 回率 $R$ 和 $F_{1}$ 值. 在机器学习领域, $A$ 是最常见的 评价指标，其计算方式比较简单，即

$$
A=\frac{\mathrm{TP}+\mathrm{TN}}{\mathrm{TP}+\mathrm{TN}+\mathrm{FP}+\mathrm{FN}}
$$

其中, $\mathrm{TP}$ 表示真正例, 表示将正类样本预测为正类 的样本数; TN 表示真负例, 表示将负类样本预测 为负类的样本数; FP 表示假正例, 表示将正类样本 预测为负类的样本数; FN 表示假负例, 表示将负 类样本预测为正类的样本数.

$R$ 又称为查全率, 也是机器学习领域常见的 评价指标之一, 其计算方式为

$$
R=\frac{\mathrm{TP}}{\mathrm{TP}+\mathrm{FP}}
$$


$F_{1}$ 值的作用是在评价模型时同时考虑 $A$ 和 $R$, 也是常用的评价指标之一, 计算方式为

$$
F_{1}=\frac{2 \times A \times R}{A+R}
$$

\section{2 样本生成过程及数据集的划分}

对于传感数据, 生成样本的主流做法如图 4 所 示, 通过设置固定大小的时间窗口在原始数据上 进行滑动获得样本. 时间窗口在原始数据上滑动 的方式有 2 种: 一种是以无重叠的方式进行滑动; 另一种是以半重叠的方式进行滑动. 2 种样本生成 方式各有优缺点, 其中, 以无重叠方式产生样本的 主要优势是样本之间无重叠, 实验结果不会造成 偏差, 但是这种方式产生的样本数量会比较少; 以 半重叠方式生成样本的优势在于能够尽可能地生 成更多的样本, 其不足之处在于重叠会带来高偏 差, 导致效果比实际要好. 在训练模型时, 需要将 数据集划分为训练集、验证集和测试集. 由于本文 采用的 3 个数据集都具有用户信息, 因此在训练模 型过程中采用交叉验证方式, 即在每次训练中留 一个用户数据用于模型的验证, 其他用户的数据 用于模型的训练. 这样做意味着每个用户的数据 都会被用作验证集, 也就是需要进行多次实验. 考虑可能存在用户在采集的数据质量低下或超预 期的情况, 所以在最后评价模型时去掉最大值和 最小值, 取平均值, 可以更加全面地评价模型的 性能.

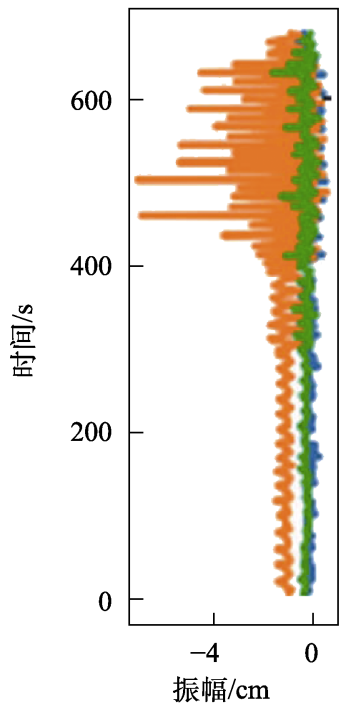

a. 原始数据

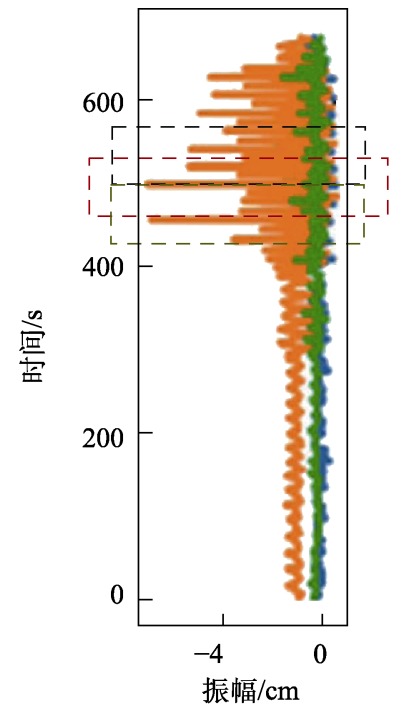

b. 获得样本
图 4 样本生成过程

\section{3 实验及结果分析}

本文在 $\mathrm{MHEALTH}^{[7]}, \mathrm{WHARF}^{[8]}$, USCHAD ${ }^{[9]}$,
Stanford-ECM Datase ${ }^{[10]}$, DATAEGO ${ }^{[11]} 5$ 个数据集 上进行实验, 并且对比了不同的方法. 为了保证方 法之间的可比较性, 本文设置相同的实验条件, 在 训练模型的过程中设置相同的实验结束条件, 即 当 loss 值小于或等于 0.2 或完成 200 次迭代训练时 结束训练模型过程. 本文采用 Adadelta 作为优化 器, 将 SoftMax 分类器作为分类器, 模型的学习率 为 0.001 , 最大迭代次数为 200 .

由表 1 可知, 与 Chen 等 ${ }^{[5]}$ 方法相比, 本文方法 在 3 个常用评价指标上都具有明显优势. 这充分说 明对于此类任务, 在网络设计中合理设计卷积核 以及网络分支的必要性. 由于 Chen 等方法在 MHEALTH 数据集上取得了极佳的效果, 精度已 经达到 91.880\%, 因此超越该方法有极大难度; 本 文方法在 MHEALTH 数据集上取得了更好的结果, 精度达到 94.080\%, 超出 Chen 等方法 $2.200 \%$, 足 以证明了本文方法的优势. 此外, 本文方法在 WHARF 数据集和 USCHAD 数据集也具有明显优 势, 但是效果并没有在 MHEALTH 数据集上提升 明显, 原因是数据集的采集方式不同, MHEALTH 数据集在采集过程中制定了严格的数据采集标准, 规范了采集动作. 但是, WHARF 数据集和 USCHAD 数据集在采集过程中没有制定如此严格 的采集标准.

表 12 种方法的性能对比

\begin{tabular}{|c|c|c|c|c|}
\hline 数据集 & 方法 & $A$ & $R$ & $F_{1}$ \\
\hline \multirow{2}{*}{ MHEALTH $^{[7]}$} & Chen 等 ${ }^{[5]}$ & 91.880 & 91.320 & 90.050 \\
\hline & 本文 & 94.080 & 94.060 & 93.070 \\
\hline \multirow{2}{*}{ WHARF $^{[8]}$} & Chen 等 ${ }^{[5]}$ & 67.050 & 42.880 & 42.600 \\
\hline & 本文 & 68.730 & 44.010 & 43.780 \\
\hline \multirow{2}{*}{$\mathrm{USCHAD}^{[9]}$} & Chen 等 ${ }^{[5]}$ & 78.830 & 74.620 & 71.370 \\
\hline & 本文 & 79.560 & 76.830 & 73.230 \\
\hline \multirow{2}{*}{$\begin{array}{l}\text { Stanford-ECM } \\
\text { Dataset }^{[10]}\end{array}$} & Chen 等 ${ }^{[5]}$ & 92.280 & 88.090 & 88.760 \\
\hline & 本文 & 93.720 & 88.810 & 90.090 \\
\hline \multirow{2}{*}{ DATAEGO ${ }^{[11]}$} & Chen 等 ${ }^{[5]}$ & 55.190 & 43.830 & 43.000 \\
\hline & 本文 & 58.170 & 45.710 & 44.680 \\
\hline
\end{tabular}

图 5 所示为 2 种方法在 Stanford-ECM Dataset 数据集 ${ }^{[10]}$ 上的实验结果, 可以看出, 本文方法在 8 个类别上取得了更好的效果，在 8 个类别上取得了 与 Chen 等 ${ }^{[5]}$ 方法相同的结果, 也有 5 个类别取得 了稍差的结果, 但从整体性能上还是有明显提升 的, 说明性能的提升并不是由于数据分布造成的, 而是方法本身的作用. 此外, 本文方法在零样本识 别任务中也有不错的表现. 其中, Wang 等 ${ }^{[20]}$ 方法 
在 CUB 数据集 ${ }^{[25]}$ 的基础上进行实验, 得到的准确 率为 $46.200 \%$, 本文方法的准确率为 $48.700 \%$ 。

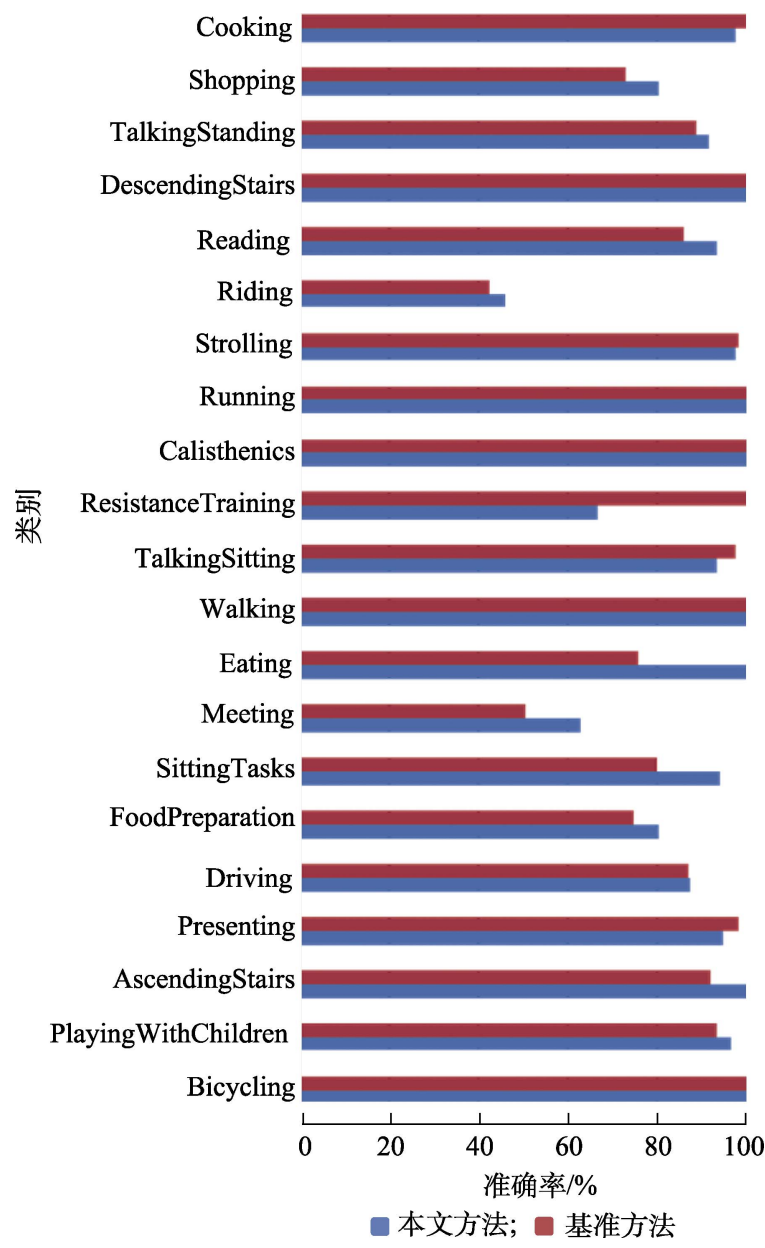

图 5 基于数据集 Stanford-ECM Dataset ${ }^{[10]}$ 的实验结果

\section{6 结 语}

本文提出了一种基于可穿戴传感器数据的人 体行为识别数据特征提取方法, 它在合理设置卷 积核的基础上引人神经网络分支结构，充分挖掘 数据的深度特征, 进一步提升了模型的检测能力. 本文在 MHEALTH ${ }^{[7]}$, WHARF ${ }^{[8]}$, USCHAD ${ }^{[9]}$, Stanford-ECM Dataset ${ }^{[10]}$, DATAEGO ${ }^{[11]} 5$ 个数据集上进 行实验，结果表明，多尺度提取数据特征的同时又 充分保持数据独立性, 对提升效果是有效的. 此外 本文将该网络引人不同的学习任务, 实验表明, 本 文方法具有较强的泛化能力, 且能够适应不同的 学习任务.

本文方法仍存在不足之处：引人分支结构增 加了算法的复杂度，增加了计算成本，这也是下一 步要解决的问题.

\section{参考文献(References):}

[1] Jordao A, Nazare A C, Sena J, et al. Human activity recognition based on wearable sensor data: a standardization of the state-of-the-art[OL]. [2020-09-30]. https://arxiv.org/abs/1806. 05226

[2] Lin T W, Liu X, Li X, et al. BMN: boundary-matching network for temporal action proposal generation[OL]. [2020-09-30]. https://arxiv.org/abs/1907.09702v1.

[3] Wang L M, Xiong Y J, Wang Z, et al. Temporal segment networks: towards good practices for deep action recognition[C] //Proceedings of European Conference on Computer Vision. Heidelberg: Springer, 2016: 22-36

[4] Lan Z Z, Zhu Y, Hauptmann A G. Deep local video feature for action recognition[C] //Proceedings of the IEEE Conference on Computer Vision and Pattern Recognition Workshops. Los Alamitos: IEEE Computer Society Press, 2017: 1219-1226

[5] Chen Y Q, Xue Y. A deep learning approach to human activity recognition based on single accelerometer[C] //Proceedings of the IEEE International Conference on Systems, Man and Cybernetics. Los Alamitos: IEEE Computer Society Press, 2015: 1488-1492

[6] Ha S, Yun J M, Choi S. Multi-modal convolutional neural networks for activity recognition[C] //Proceedings of the International Conference on Systems, Man, and Cybernetics. Los Alamitos: IEEE Computer Society Press, 2016: 3017-3022

[7] Banos O, Garcia R, Holgado-Terriza J A, et al. mHealthDroid: a novel framework for agile development of mobile health applications[C] //Proceedings of International Workshop on Ambient Assisted Living. Heidelberg: Springer, 2014: 91-98

[8] Bruno B, Mastrogiovanni F, Sgorbissa A. Wearable inertial sensors: applications, challenges, and public test benches[J]. IEEE Robotics \& Automation Magazine, 2015, 22(3): 116-124

[9] Lockhart J W, Weiss G M, Xue J C, et al. Design considerations for the WISDM smart phone-based sensor mining architecture[C] //Proceedings of the 5th International Workshop on Knowledge Discovery from Sensor Data. New York: ACM Press, 2011: 25-33

[10] Nakamura K, Yeung S, Alahi A, et al. Jointly learning energy expenditures and activities using egocentric multimodal signals[C] //Proceedings of the IEEE Conference on Computer Vision and Pattern Recognition. Los Alamitos: IEEE Computer Society Press, 2017: 6817-6826

[11] Possas R, Caceres S P, Ramos F. Egocentric activity recognition on a budget $[\mathrm{C}] / /$ Proceedings of the IEEE Conference on Computer Vision and Pattern Recognition. Los Alamitos: IEEE Computer Society Press, 2018: 5967-5976

[12] Lu Zhiyuan. Research on some key technologies of wearable health monitoring and human-computer interaction application[D]. Hefei: University of Science and Technology of China, 2014(in Chinese)

(路知远. 穿戴式健康监护及人机交互应用中若干关键技术 研究[D]. 合肥: 中国科学技术大学, 2014)

[13] Wang Liang. Research on wearable sensor network based human activity recognition technologies[D]. Nanjing: Nanjing University, 2014(in Chinese) (汪亮. 基于可穿戴传感器网络的人体行为识别技术研究 
[D]. 南京: 南京大学, 2014)

[14] Ru Chenguang. Research on sensor-based human behavior recognition technology[D]. Hangzhou: Zhejiang University, 2016(in Chinese)

(茹晨光. 基于传感器的人体行为识别技术研究 [D]. 杭州: 浙江大学, 2016)

[15] Amato G, Bacciu D, Broxvall M, et al. Robotic ubiquitous cognitive ecology for smart homes[J]. Journal of Intelligent \& Robotic Systems, 2015, 80: 57-81

[16] Ohnishi K, Hidaka M, Harada T. Improved dense trajectory with cross streams[C] //Proceedings of the 24th ACM International Conference on Multimedia. New York: ACM Press, 2016: 257-261

[17] Wang H, Klaser A, Schmid C, et al. Action recognition by dense trajectories[C] //Proceedings of the IEEE Conference on Computer Vision and Pattern Recognition. Los Alamitos: IEEE Computer Society Press, 2011: 3169-3176

[18] Simonyan K, Zisserman A. Two-stream convolutional networks for action recognition in videos[C] //Proceedings of the 27th International Conference on Neural Information Processing Systems. Cambridge: MIT Press, 2014: 568-576

[19] Tran D, Bourdev L, Fergus R, et al. Learning spatio-temporal features with 3D convolutional networks[C] //Proceedings of the IEEE International Conference on Computer Vision. Los Alamitos: IEEE Computer Society Press, 2015: 4489-4497
[20] Wang L M, Xiong Y J, Wang Z, et al. Temporal segment networks: towards good practices for deep action recognition[C] //Proceedings of European Conference on Computer Vision. Heidelberg: Springer, 2016: 22-36

[21] Ding Chongyang, Liu Kai, Li Guang, et al. Spatio-temporal weighted posture motion features for human skeleton action recognition research[J]. Chinese Journal of Computers, 2020, 43(1): 29-40(in Chinese)

(丁重阳, 刘凯, 李光, 等. 基于时空权重姿态运动特征的人 体骨架行为识别研究 [J]. 计算机学报, 2020, 43(1): 29-40)

[22] Antoniou A, Storkey A, Edwards H. Data augmentation generative adversarial networks[OL]. [2020-09-30]. https://arxiv. org/abs/1711.04340v3

[23] Meng W H, Zhang S, Yao X D, et al. Biomedia ACM MM grand challenge 2019: using data enhancement to solve sample unbalance[C] //Proceedings of the 27th ACM International Conference on Multimedia. New York: ACM Press, 2019: 2588-2592

[24] Wang D H, Li Y N, Lin Y T, et al. Relational knowledge transfer for zero-shot learning[C] //Proceedings of the 30th AAAI Conference on Artificial Intelligence. Palo Alto: AAAI Press, 2016: 2145-2151

[25] Wah C, Branson S, Welinder P, et al. The caltech-UCSD birds-200-2011 dataset[OL]. [2020-09-30]. https://core.ac. uk/display/4892191 